\title{
Tuning the Properties of Nanoceria by Applying Force: Stress - Induced Ostwald Ripening
}

\author{
Francesco Caddeo $^{\mathrm{a}}$, Anna Corrias ${ }^{\mathrm{a}}$, Dean C. Sayle $\mathrm{a}^{\mathrm{a}}$ \\ a School of Physical Sciences, Ingram Building, University of Kent, Canterbury CT27NH, \\ United Kingdom
}

\begin{abstract}
The ionic conductivity and activity of a material, such as ceria, is central to its exploitation in applications such as fuel cells or catalysis. Grain Boundaries (GB) influence profoundly the ionic conductivity and activity of a material. Accordingly, the ability to control GB concentrations is pivotal to these applications. Here, we show that Oswald ripening can be induced by applying uniaxial force. In particular, grain-boundaries are purged to facilitate single nanocrystals from polycrystalline precursors. Our simulations thus predict mechanisms for strain-tunable properties.
\end{abstract}

\section{INTRODUCTION}

Nanoceria enjoys applications including: catalysis ${ }^{1}$, solid oxide fuel cells ${ }^{2}$, solar cells $s^{3}$, oxygen storage material $s^{4}$, nanomedicine ${ }^{5}$, and chemical mechanical planarization ${ }^{6}$. The nature of nanoceria influences its properties and therefore synthetic routes have been devised to control the size, shape and microstructure of ceria nanomaterials: techniques such as hydrothermal ${ }^{7}$ and solvothermal ${ }^{8}$ crystallization, precipitation ${ }^{9}$ and liquid phase spray pyrolysis ${ }^{10}$ are used to selectively synthesize ceria nanoparticles with cuboidal ${ }^{11}$, polyhedral $^{12}$ and spherical morphologies ${ }^{10}$, ceria nanorods ${ }^{13}$ and nanotubes ${ }^{14}$ and nanoceria with 3-dimensional mesoporous architectures ${ }^{15}$.

Central to the wide variety of applications of ceria is the ability of the material to store and release oxygen. Such oxygen storage capacity (OSC) bestows exemplary catalytic properties upon the material ${ }^{16}$. However, oxygen released from the surface of the material must be replenished and therefore the oxygen 
ion conductivity of the material impacts upon its OSC; the oxygen ion conductivity of ceria is sufficiently high to enable ceria to act as a viable electrolyte in solid oxide fuel cells. It is not surprising therefore that considerable efforts have been directed at controlling (increasing) the ionic conductivity of the material ${ }^{17}$. However, grain-boundaries, GB, manifest as obstacles to high ionic conductivity and can reduce the conductivity by orders of magnitude ${ }^{18}$. Typically, oxygen ion conductivity is vacancy driven ${ }^{19}$ and therefore sensitive to local environment. Grain-boundaries change the local structure and stifle the ability of oxygen ions to traverse the interface; defect segregation to $G B$ regions compound this effect ${ }^{18}$. Grain boundaries also play a crucial role with respect to the activity of a material. Consolidation of nanocrystals with the formation of grain boundaries can lead to an increase in the catalytic activity of a material by one order of magnitude 20

Clearly, the ability to control microstructural features, such as GB, will enable one to optimise the ionic conductivity. Here, we explore how mechanical stress may be used to purge GB from nanoceria.

Ceria nanomaterials have been widely studied in terms of morphology, surfaces exposed and reactivity. Conversely, the mechanical properties have received much less attention even though they are very different from the parent bulk material $^{21}$ and are central to applications such as chemical mechanical planarization $^{10}(\mathrm{CMP})^{\mathrm{a}}$. Experimentally, nanoindentation, in-situ Transmission Electron Microscopy (TEM) and Atomic Force Microscopy (AFM) have been used to determine the relationships between the mechanical properties and structures of nanomaterials ${ }^{22}, 23,24,25$. However, the time-resolved structural characterisation of strained materials at the nanoscale is challenging experimentally ${ }^{26}$ and therefore most studies consider nanomaterials with dimensions of $100 \mathrm{~nm}$ or more; a review is given in reference ${ }^{27}$. Simulation can therefore provide unique insight for experiment: small systems are computationally more tractable than large systems and it is possible to deconvolute and explore how each structural feature influences the mechanical properties.

In this work, we calculate the mechanical properties of ceria nanoparticles with cuboidal, polyhedral and spherical shapes, comprising rich microstructures, by simulating the particles under uniaxial compression. Moreover, nanoceria is easily reduced ${ }^{28}$ and therefore we also calculate the mechanical properties of partially reduced ceria - $\mathrm{CeO}_{1.9}$.

\footnotetext{
${ }^{a}$ It is estimated that the global production of nanoceria for CMP process is ca. 170 tons per year
} 


\section{METHODS}

Potential models All simulations presented in this study are based on the Born model of the ionic solid. The energy of the system, $Q_{i}$, is given by:

$$
E\left(r_{i j}\right)=\sum_{i j} \frac{Q_{i} Q_{j}}{4 \pi \varepsilon_{o} r_{i j}}+\sum_{i j} A \exp \left(\frac{-r_{i j}}{\rho}\right)-C r_{i j}^{-6}
$$

where the first term represents the Coulombic interaction of a pair or ions and the second term is in the Buckingham form. The model parameters used to describe $\mathrm{CeO}_{2}$ nanoparticles are shown in Table $1^{29,}{ }^{30}$. Formal ionic charges are employed; a more detailed discussion concerning the use of this potential model is found in supporting information (Discussion S1, Discussion S2)

Simulation code The simulations where performed using the DL_POLY $\operatorname{code}^{31}$; the user manual provides comprehensive analytical description and discussion of the molecular dynamics simulations, force fields, boundary conditions and algorithms used in these simulations.

Table 1 Potential parameters used to describe the interactions between ions comprising the nanoparticles.

\begin{tabular}{|c|c|c|c|c|c|}
\hline Atom $\mathrm{i}$ & Atom $\mathrm{j}$ & $\mathrm{A} / \mathrm{eV}$ & $\rho / \AA$ & C / EvÅ & Cut - off / $\AA$ \\
\hline 0 & 0 & 22764.30 & 0.149 & 27.89 & 10.0 \\
\hline 0 & $\mathrm{Ce}(\mathrm{IV})$ & 1986.83 & 0.351 & 20.40 & 10.0 \\
\hline 0 & $\mathrm{Ce}(\mathrm{III})$ & 1731.62 & 0.364 & 14.43 & 10.0 \\
\hline $\mathrm{Ce}(\mathrm{IV})$ & $\mathrm{Ce}(\mathrm{IV})$ & Set to zero & & & \\
\hline $\mathrm{Ce}(\mathrm{IV})$ & $\mathrm{Ce}(\mathrm{III})$ & Set to zero & & & \\
\hline $\mathrm{Ti}(\mathrm{IV})$ & 0 & 760.47 & 0.388 & 0.00 & 10.0 \\
\hline Atom & & Mass / amu & & Charge / e & \\
\hline 0 & & 16.0 & & -2.0 & \\
\hline $\mathrm{Ce}(\mathrm{IV})$ & & 140.12 & & +4.0 & \\
\hline $\mathrm{Ce}(\mathrm{III})$ & & 140.12 & & +3.0 & \\
\hline $\mathrm{Ti}(\mathrm{IV})$ & & 47.867 & & +4.0 & \\
\hline
\end{tabular}


Atomistic model generation Full atomistic models of ceria nanoparticles with cuboidal, polyhedral and spherical shapes were generated using simulated amorphisation and re-crystallization ${ }^{10}$. This technique facilitates models that are in quantitative structural accord with experiment and enables microstructural features, such as grain-boundaries and dislocations that will influence the mechanical properties of the nanomaterial, to be captured within the models ${ }^{32}$. Analogous to experiment, the spontaneous evolution of the nucleating seed and subsequent crystallization drive the final structure of the model nanoparticles rather than the simulator imposing structure (perhaps erroneously) upon the model. Previously, we showed that by changing the temperature of crystallization, single-crystal or polycrystalline nano-particles could be generated 33

All the images presented are representations of the atoms positions and are not schematic; analyses of the structures were performed using $\mathrm{VMD}^{34}$ and Material Studio.

Ceria nanocube To generate an atomistic model of a ceria nanocube, Figure 1a, a crystal of $\mathrm{CeO}_{2}$ comprising 20736 atoms (6912 Ce atoms, $13824 \mathrm{O}$ atoms) was melted while holding fixed fluorite - structured inner core with cuboidal morphology comprising 12000 atoms (4000 Ce, 8000 O) and performing constant volume MD simulation at $8000 \mathrm{~K}$ for 50 ps. Crystallization was then performed applying constant volume MD simulation at $1500 \mathrm{~K}$ for $300 \mathrm{ps.} \mathrm{During}$ the crystallisation, all the atoms (including the inner core) were allowed to move to reduce any bias in the structural evolution of the model. The strategy of using a cubic seed was to avoid the natural tendency of ceria to crystallize with truncated octahedral shape, exposing low energy $\{111\}$ surfaces ${ }^{35}$. Once the nanocube had formed, constant volume MD simulation at $3750 \mathrm{~K}$ was performed for $27 \mathrm{~ns}$; the evolution of an octahedral morphology was not observed, rather the cuboidal morphology persisted, indicating that the morphology is structurally stable in accord with experiment. We note that $27 \mathrm{~ns}$ is a very short time period - more than 12 orders of magnitude shorter than experimental annealing conditions. However, simulated annealing at elevated temperatures (3750K) helps accelerate the dynamics and mitigate the difference in timescales. Finally, the structure was cooled by performing constant volume MD simulation at $1 \mathrm{~K}$ for 50 ps. 


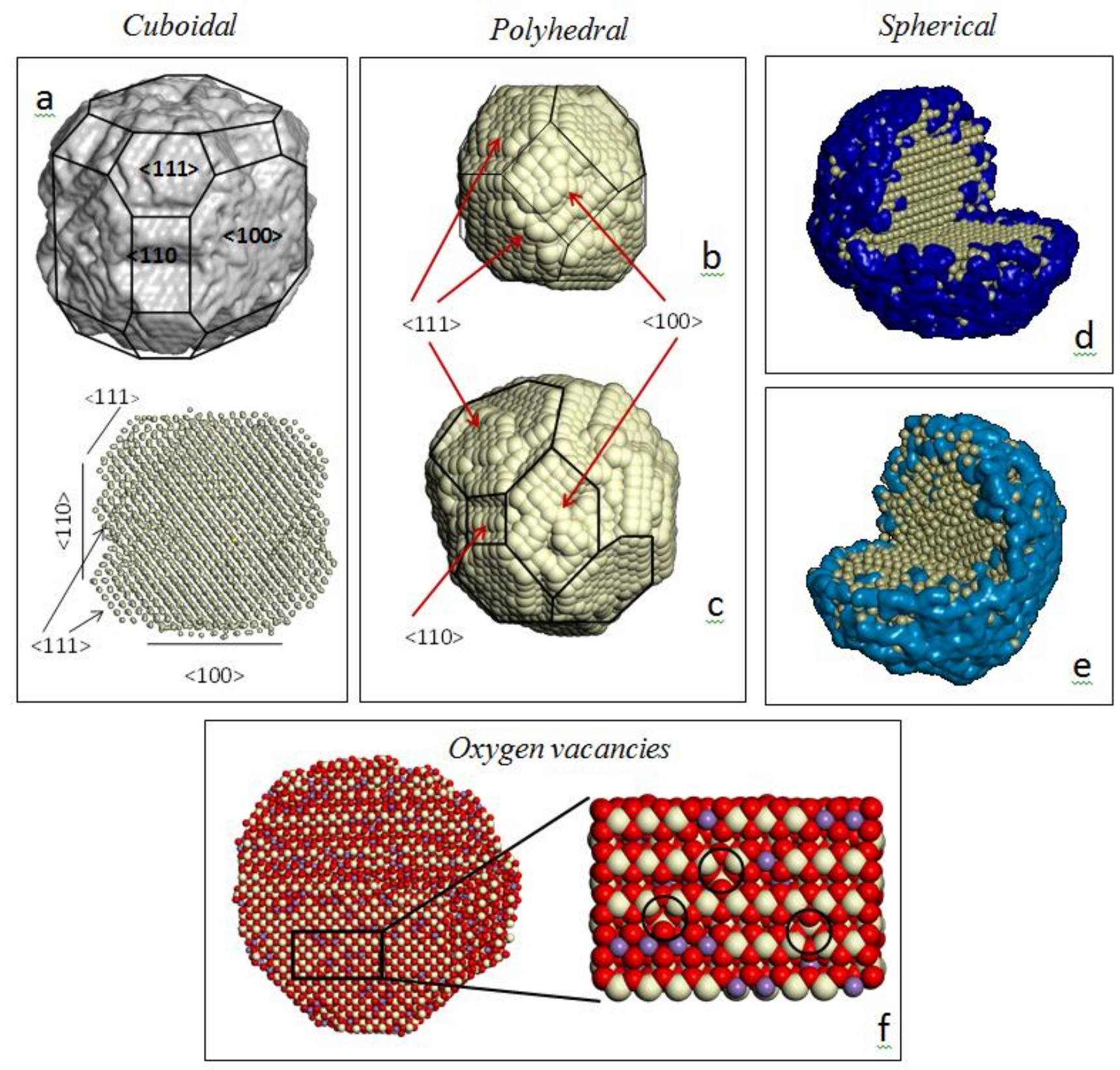

Figure 1 Full atomistic models of $\mathrm{CeO}_{2}$ NanoParticles, NP: (a) $8 \mathrm{~nm}$ nanocube, (b) $6 \mathrm{~nm}$ polyhedral NP, (c) $8 \mathrm{~nm}$ polyhedral NP, (d) $8 \mathrm{~nm}$ core-shell (single crystal core) NP, (e) $8 \mathrm{~nm}$ core-shell (polycrystalline core) NP, (f) Partially reduced $8 \mathrm{~nm}$ polyhedral NP $\left(\mathrm{CeO}_{1.9}\right)$ showing the presence of oxygen vacancies (circled). A section has been cut from both spherical particles to reveal the inner $\mathrm{CeO}_{2}$ core. $\mathrm{Ce}^{4+}$ ions are represented by white spheres, $\mathrm{O}^{-2}$ ions by red spheres and $\mathrm{Ce}^{3+}$ ions by purple spheres. Surface rendering is used to show the outer $\mathrm{TiO}_{2}$ shell.

Polyhedral nanoparticles Two ceria nanoparticles with polyhedral shape were generated following methods reported in ref ${ }^{35}$. In particular, nanoparticles of $\mathrm{CeO}_{2}$ comprising 7761 and 18849 atoms were amorphised and recrystallized, resulting in two polyhedral nanoparticles with diameters of ca. $6 \mathrm{~nm}$ and $8 \mathrm{~nm}$ respectively (Figure 1c) 
Oxygen vacancies The reduced $\left(\mathrm{CeO}_{1.9}\right)$ model nanoparticle was generated by removing $5 \%$ (628 ions) of the oxygen ions from random positions within the $8 \mathrm{~nm}$ polyhedral ceria particle; charge neutrality was maintained by changing the charge state of two cerium ions from 4+ to 3+ for every oxygen vacancy (1256 in total). The nanoparticle was then equilibrated by performing MD simulation, using an NVT ensemble, at $2000 \mathrm{~K}$ for $2.5 \mathrm{~ns}$, enabling the oxygen vacancies to locate to low-energy positions.

We note that oxygen vacancies, initially generated on the surface of the nanoparticle, moved to sub-surface positions in accord with previous findings ${ }^{36}$.

Spherical $\mathrm{CeO}_{2}-\mathrm{TiO}_{2}$ Core-Shell nanoparticles To generate spherical nanoparticles, we employed a method reported previously in ref ${ }^{10}$. In particular, a nanoparticle of $\mathrm{CeO}_{2}$ comprising 15972 atoms was taken and $\mathrm{Ce}^{4+}$ ions within the outer region of the particle were replaced by $\mathrm{Ti}^{4+}$. The particle was then amorphised and re-crystallized using MD simulation. The temperature was chosen carefully such that the inner $\mathrm{CeO}_{2}$ core crystallizes while the outer $\mathrm{TiO}_{2}$ shell stays molten. A molten material will minimise its surface energy by adopting a spherical morphology, thus facilitating a spherical nanoparticle. The strategy exploits the fact that the melting point of $\mathrm{CeO}_{2}$ is higher than $\mathrm{TiO}_{2}$ and therefore at a particular temperature, the $\mathrm{CeO}_{2}$ crystallises while the $\mathrm{TiO}_{2}$ remains molten. Two nanoparticles were generated, the first comprises a singlecrystal ceria core, whereas the ceria core of the second nanoparticle was polycrystalline (Figure 1d, Figure 1e). In particular, careful choice of recrystallisation temperature enables the evolution of either a single crystal or a polycrystalline $\mathrm{CeO}_{2}$ core ${ }^{37}$.

Uniaxial Compression The mechanical properties are calculated by simulating the nanoparticles under uniaxial compression. In particular, a nanoparticle is positioned at the center of the simulation cell and two planes of atoms (acting as an "anvil") inserted perpendicular to the compression direction, Figure 2. The nanoparticle is then equilibrated by performing constant pressure (NPT) MD simulation, imparting zero pressure, at 300K for 100 ps. This preliminary step releases any residual stress emanating from the crystallization of the nanoparticle or from the addition of the "anvils".

Uniaxial compression is simulated by moving the planes of atoms, comprising the anvils, towards one another under NVT MD simulation at $300 \mathrm{~K}$. In particular, the anvil is moved by $0.01 \%$ of the length of the simulation cell followed by MD 


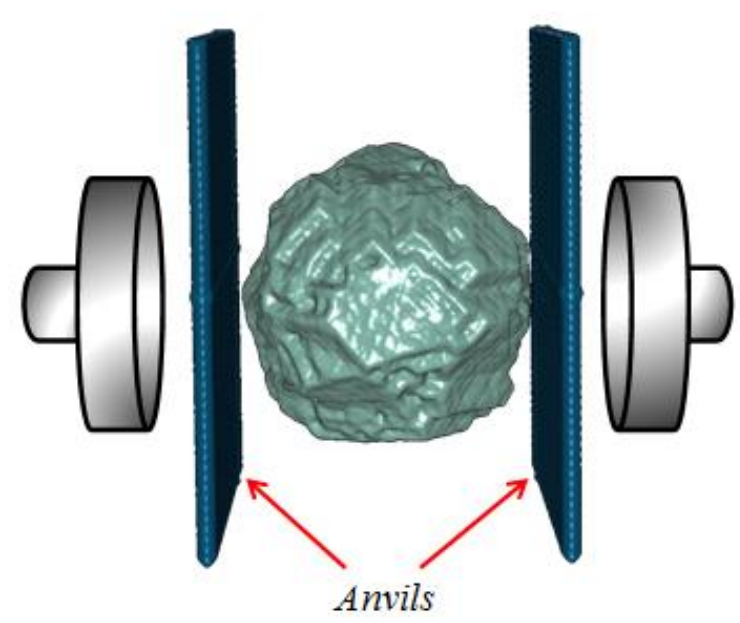

Figure 2 Schematic describing the method for imparting uniaxial stress upon the nanoparticles

simulation performed for $100 \mathrm{ps}$ to give an average strain rate of $0.07 \mathrm{~m} / \mathrm{s}$. with an overall simulation time of $511 \mathrm{~ns}$.

The strain is expressed as:

Strain $=\left(L_{O}-L_{F}\right) / L_{0}$

where $L_{o}$ and $L_{F}$ correspond to the original and final length of the nanoparticle along the direction of imposed stress respectively; as we only consider uniaxial compression, the strains are all reported as positive values.

A similar approach was used previously in order to calculate the mechanical properties of ceria nanorods ${ }^{38}$; however, in that work, the coordinates of all the atoms of the nanorod were scaled at the same time. Here, the nanoparticles are compressed by the repulsive interactions with the plane of atoms (anvils) between them, enabling the strain to travel from the surface to the bulk of the nanoparticles, in accord with how the real material would respond under uniaxial compression.

\section{RESULTS}

Here, we report upon the atomistic structures of the nanoparticles, their mechanical properties, and associated strain-induced deformation mechanisms.

\section{Atomistic structures}

Nanocube The atomistic structure of the ceria nanocube is shown in Figure 1 a. The predominant $\{100\}$ surfaces are truncated at the edges and corners with 
$\{110\}$ and $\{111\}$ facets; the $\{110\}$ surfaces are also in part $\{111\}$-faceted surface in accord with the real material. In particular, corrugation of the $\{110\}$ surface emanating from faceting into $\{111\}$ - has been indicated in the literature, together with the truncation of the corners into $\{111\}$ faces $^{39}$.

Polyhedral nanoparticles Without the "shape directing" crystalline seed, used to generate the nanocube, the (simulated) crystallisation facilitated polyhedral nanoparticles, which expose thermodynamically stable $\{111\}$ surfaces truncated by $\{100\}$. The model nanoparticles are shown in Figure $1 \mathrm{~b}(6 \mathrm{~nm} N \mathrm{~N})$ and Figure $1 \mathrm{c}(8 \mathrm{~nm} \mathrm{NP})$ and are in structural accord with experiment ${ }^{40}$. Both nanoparticles are polycrystalline; the grain boundaries evolve 'naturally' during the simulated crystallization. A Coincidence Site Lattice (CSL) theory ${ }^{41}$ was used to characterise the grain boundaries within the nanoparticles. In particular, graphical analysis of the $6 \mathrm{~nm}$ NP reveals a $\Sigma 3$ twin grain boundary. This special, highly symmetric grain boundary, also called a coherent twin boundary, can be described as a $60^{\circ}$ rotation along $<111>$ of one crystal grain with respect to the other. It has been demonstrated that this grain boundary is energetically stable ${ }^{25}$ in ceria. Analysis of the GB structures in the $8 \mathrm{~nm}$ NP reveal a $\Sigma 11$ grain boundary, which can be described as a rotation of one of the crystal grains by 1250 along the $<311>$ crystal plane.

Oxygen vacancies Analysis of the reduced ceria nanoparticle, $\mathrm{CeO}_{1.9}$, reveals a polyhedral morphology with $\{111\}$ facets truncated by $\{100\}$ which is commensurate with the fully oxidised particle. A section of the nanoparticle, viewed along $\langle 110\rangle$, is shown in Figure 1f; the oxygen vacancies are highlighted by the black rings.

Spherical Core-Shell Nanoparticles Analysis of the two spherical coreshell ceria nanoparticles reveals one comprises a single crystalline inner ceria core, Figure 1d, whereas the ceria core of the other is polycrystalline, Figure 1e; the amorphous shell of $\mathrm{TiO}_{2}$ prevents the formation of a faceted surface facilitating the spherical morphology

Mechanical properties The calculated mechanical properties, derived from the simulated stress-strain curves, Figure 4, are shown in Figure 3 and Table 2. 

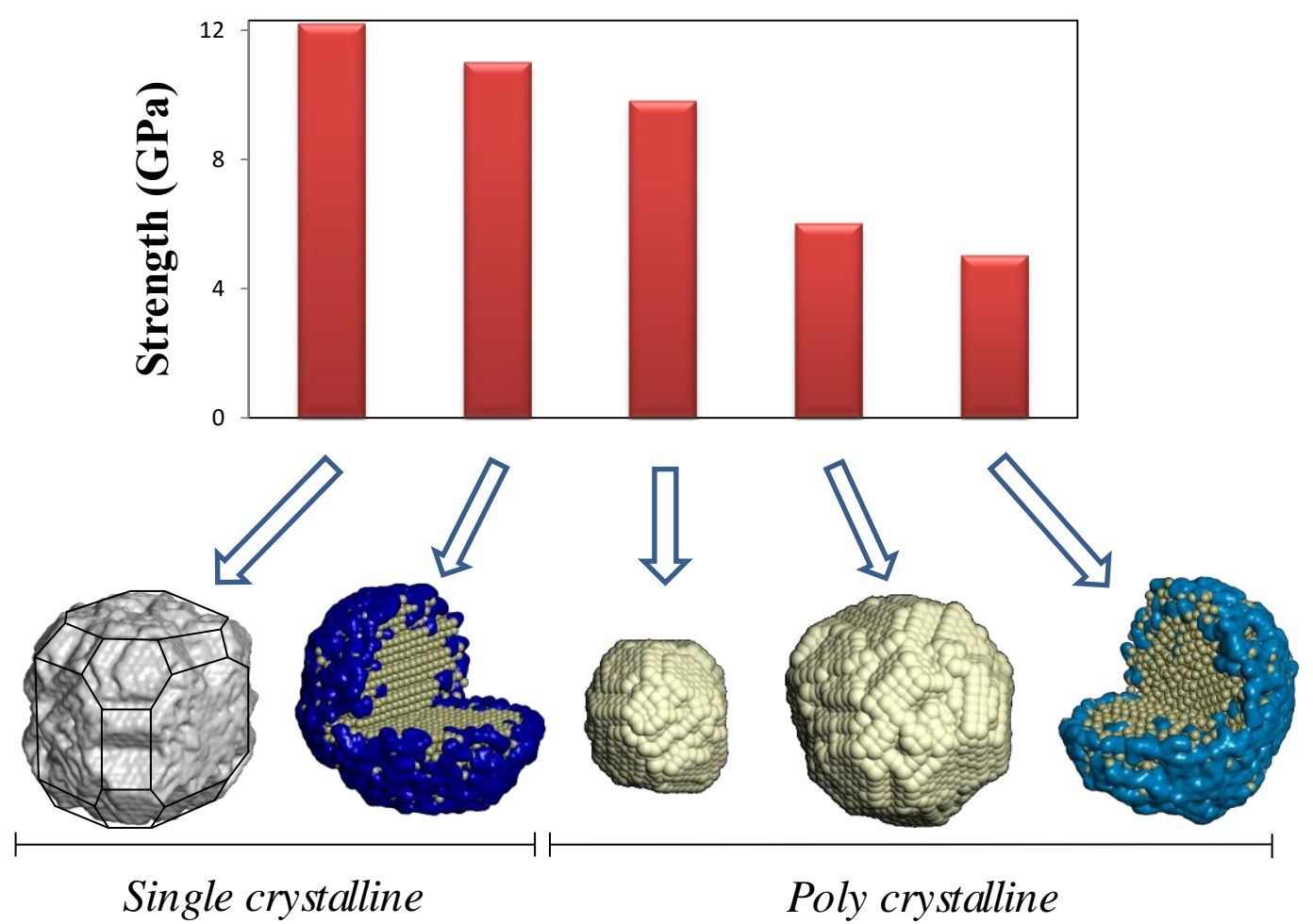

Figure 3 Compressive strengths of each of the ceria nanoparticles

Nanocube The nanocube has the highest yield stress of all the nanoparticles considered and reaches $12 \mathrm{GPa}$ at 0.20 strain, Figure 4 a (point A). The nanocube deforms elastically, with a non-linear slope, up to 0.20 strain and then deforms plastically, via a crystalline-to-amorphous transition, Figure S1 (a-d) (supporting information). A screw dislocation, terminating at a grain boundary, provides a vehicle for further plastic deformation, Figure S1 (e-j), supporting information. In particular, Figure S1(j) shows the structure of a strain-induced low-angle grainboundary

$6 \mathrm{~nm}$ polyhedral nanoparticle The 'small' nanoparticle is weaker than the nanocube and deforms elastically up to a strain of 0.16 , with a yield strength of $10 \mathrm{GPa}$, Figure $4 \mathrm{~b}$, point $\mathrm{A}$. The nanoparticle then suffers plastic deformation, driven by lattice slip along the $\Sigma 3$ grain boundary; a screw dislocation evolves at higher strains, Figure 4(b), Point B. Plastic deformation mechanisms are shown in Figure S2, supporting information.

$8 \mathrm{~nm}$ polyhedral nanoparticle Increasing the size of the polyhedral nanoparticle from $6 \mathrm{~nm}$ to $8 \mathrm{~nm}$, reduces its yield strength from $10 \mathrm{GPa}$ to $6 \mathrm{GPa}$, Figure 4c. Moreover, plastic deformation, driven via slip along the $\Sigma 11$ boundary plane, Figure 5, occurs at lower strains: $0.10(8 \mathrm{~nm} \mathrm{NP})$ compared to $0.16(6 \mathrm{~nm}$ 
NP). The structural complexity of the grain-boundary network is shown in Figure S5. In particular, the images reveal that the oxygen sublattice is heavily disordered at the grain-boundary region.

$8 \mathrm{~nm}$ polyhedral nanoparticle-oxygen vacancies Nanoceria is rarely fully oxidised and depending upon its environment (notably oxygen partial pressure) the particle will likely be partially reduced. It is therefore intriguing to explore how reduced ceria, $\mathrm{CeO}_{1.9}$, which comprises $5 \%$ oxygen vacancies and $20 \%$ of the $\mathrm{Ce}^{4+}$ ions reduced to $\mathrm{Ce}^{3+}$, impacts upon its mechanical properties. The stressstrain curves for the fully oxidised, $\mathrm{CeO}_{2}$, and partially reduced, $\mathrm{CeO}_{1.9}$, ceria are superimposed in Figure 4(c). Inspection of the figure reveals that the partially reduced particle is about $16 \%$ weaker. The gradient of the stress-strain curve pertaining to the elastic deformation region, which is indicative of the elastic modulus, is also $34 \%$ lower compared to the fully oxidised nanoparticle, Table 2.

Core-Shell nanoparticle - single crystal The core-shell nanoparticle deforms elastically up to 0.13 strain, Figure $4(d)$, point A. This is followed by amorphisation of the ceria at the interfacial region, Figure S3 (e-g). The ultimate yield strength of $11 \mathrm{GPa}$ is reached at 0.34 strain, Figure $4(\mathrm{c})$, point $\mathrm{B}$ and Figure S3(j)

Core-Shell nanoparticle-polycrystal The core-shell nanoparticle with a polycrystalline ceria core is, perhaps unsurprisingly, weaker compared to its single crystal counterpart; the yield strength at 0.10 strain is only $2.3 \mathrm{GPa}$, Figure $4(d)$, point $A$ (red trace). Plastic deformation, driven by grain-boundary slip occurs at uniaxial stresses above 2.3GPa; deformation mechanisms are presented in Figure S4, supporting information. 
Table 2 Mechanical properties calculated for each of the ceria nanoparticles and literature values for the bulk modulus obtained experimentally and by DFT calculations.

\begin{tabular}{|c|c|c|c|c|c|}
\hline Nanoparticle & $\begin{array}{l}\text { Diameter } \\
\mathrm{nm}\end{array}$ & $\begin{array}{l}\text { Area } \\
\mathrm{nm}^{2}\end{array}$ & $\begin{array}{l}\text { Yield } \\
\text { Strength } \\
\text { GPa }\end{array}$ & Yield Strain & $\begin{array}{l}\text { Elastic } \\
\text { Modulus } \\
\text { GPa }\end{array}$ \\
\hline Cuboidal & 8 & 64 & 12 & 0.20 & 157 \\
\hline 6nm Polyhedral & 6 & 28 & 10 & 0.16 & 84 \\
\hline 8nm Polyhedral & 8 & 50 & 6 & 0.10 & 62 \\
\hline $\mathrm{CeO}_{1.9} 8 \mathrm{~nm} \mathrm{NP}$ & 8 & 50 & 5 & 0.13 & 41 \\
\hline $\mathrm{TiO}_{2}-\mathrm{CeO}_{2} \mathrm{NP}$ & 8 & 50 & 11 & 0.13 & 85 \\
\hline $\mathrm{TiO}_{2}-\mathrm{CeO}_{2} \mathrm{NP}(\mathrm{GB})$ & 8 & 50 & 5 & 0.10 & 20 \\
\hline Literature & \multicolumn{2}{|c|}{ Bulk modulus (GPa) } & \multicolumn{2}{|l|}{ Method } & Reference \\
\hline $\mathrm{CeO}_{2}$ bulk & \multicolumn{2}{|l|}{$112-174$} & \multicolumn{2}{|c|}{ Small punch (SP) } & 48,49 \\
\hline $\mathrm{CeO}_{2}$ bulk & \multicolumn{2}{|l|}{$190-264$} & \multicolumn{2}{|c|}{ Nano indentation (ND) } & 46 \\
\hline $\mathrm{CeO}_{2}-12 \mathrm{~nm}$ & \multicolumn{2}{|l|}{$282-289$} & \multicolumn{2}{|l|}{$A D X D$} & 47 \\
\hline $\mathrm{CeO}_{2}-6 \mathrm{~nm}$ & \multicolumn{2}{|l|}{180} & \multicolumn{2}{|c|}{ High Pressure XRD } & 50 \\
\hline $\mathrm{CeO}_{2}$ & \multicolumn{2}{|l|}{249} & \multicolumn{2}{|c|}{ DFT calculations } & 51 \\
\hline
\end{tabular}



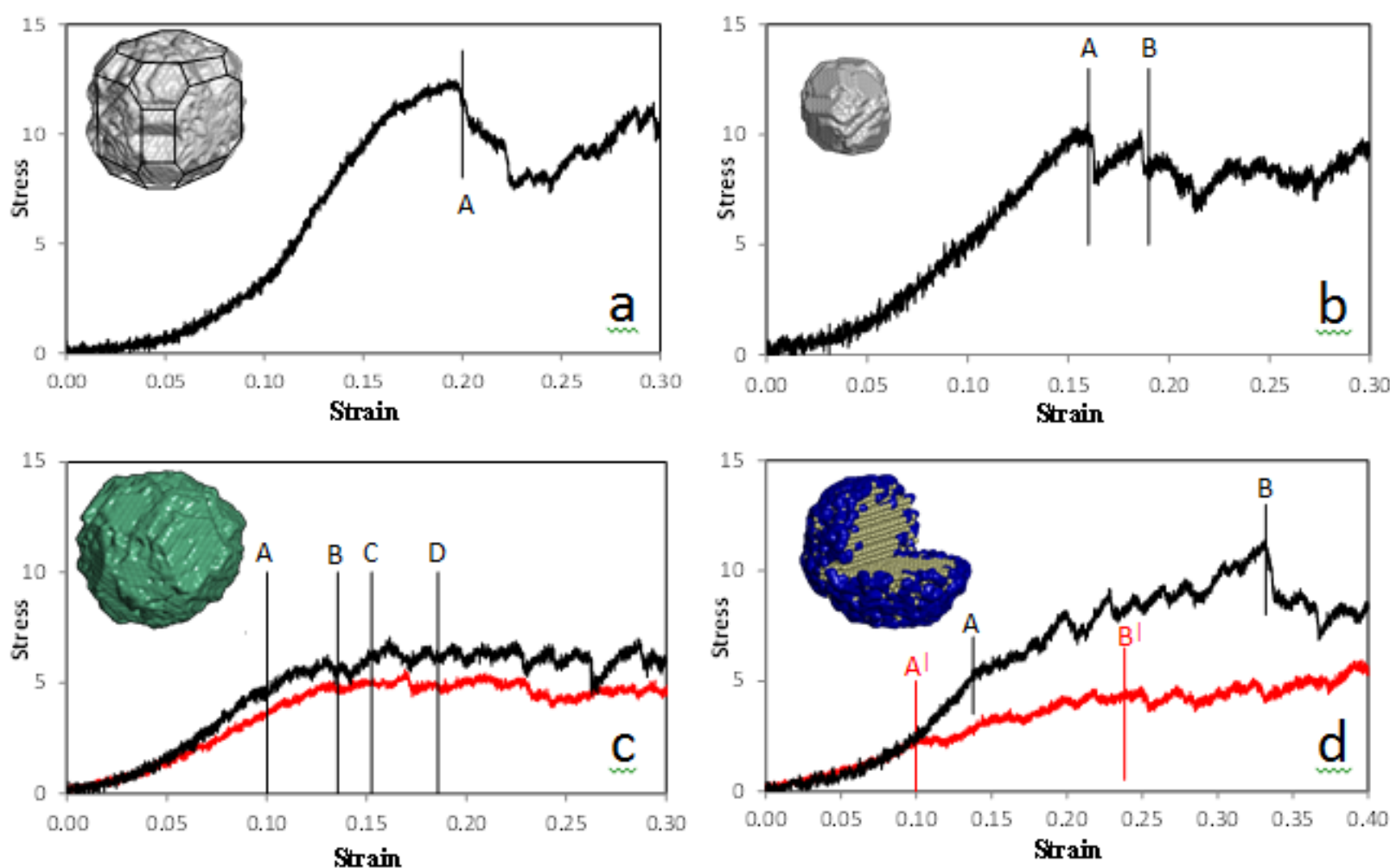

Figure 4 Stress - strain curves calculated for the model ceria nanoparticles under uniaxial compression: (a) $8 \mathrm{~nm}$ nanocube, (b) 6nm polyhedral NP, (c) 8nm polyhedral NP; fully oxidised $\mathrm{CeO} 2$ (black trace) and reduced CeO1.9 (red trace), (d) $8 \mathrm{~nm}$ Core - Shell nanoparticles with single - crystal (black trace) and polycrystalline (red trace) ceria cores.

\section{DISCUSSION}

Strain - Induced Ostwald Ripening

Inspection of Figure 5 reveals that, under uniaxial compression, one grain grows in size at the expense of the other, which is indicative of Oswald ripening ${ }^{42}$. In particular, the top grain (yellow) grows by consuming the bottom grain (blue). The images, Figure 5 (b-e) correlate with points A-D on the stress-strain curve, Figure 4c, which reveals that Oswald ripening increases the yield stress of the nanoparticle from 4.5 to $7 \mathrm{GPa}$.

Our simulations reveal that stress-induced Ostwald Ripening is facilitated by lattice slip along the $\Sigma 11$ boundary. The grain boundary is thus purged from the nanoparticle to facilitate the formation (crystal growth) of a single crystal. It has been shown that grain boundaries can dominate the ionic transport properties of the materials ${ }^{18}$. Accordingly, our simulations predict that one can influence the transport properties of a nanomaterial by mechanically tuning its grain-boundary content. 
A review by Guo and Waser ${ }^{43}$ details that 'grain-boundary conductivities of acceptor-doped zirconia and ceria are at least two orders of magnitude lower than the corresponding bulk values, depending on temperature and dopant level. Furthermore, a review by Landau and co-workers details the importance of engineering the content of grain boundaries in order to impact on the catalytic activity of a material. The consolidation of nanocrystals with the formation of grain boundary areas can lead to an increase of the chemical reactivity by one order of magnitude ${ }^{44}$; our simulations reveal that grain-boundaries can also be induced via strain, Figure $\mathrm{S} 1(\mathrm{~g})$, supporting information. The ability to mechanically engineer the grain-boundary content of a material therefore impacts upon technological important areas such as solid oxide fuel cells $s^{45}$ and catalysis $^{44}$.

Models with full microstructure The simulations reveal that the compressive strength of ceria nanoparticles depends critically upon the presence of extended defects such as grain boundaries and dislocations. In particular, the spherical nanoparticle without grain-boundaries has a $400 \%$ higher elastic modulus, and $200 \%$ higher yield strength. This epitomises the need to include microstructural features within the models.

Wang at al. ${ }^{46}$ determined the elastic modulus of fully oxidised and partially reduced ceria by performing nanoindentation tests at low oxygen partial pressure. They found that the elastic modulus of ceria decreases by $28 \%$ when the oxygen partial pressure of the environment is reduced to $10^{-25} \mathrm{~atm}$. This has been attributed to the formation of oxygen vacancies within the fluorite crystal structure. We found that the elastic modulus of the partially reduced nanoparticle, $\mathrm{CeO}_{1.9}$, is about $30 \%$ lower compared to the fully oxidised NP, in line with the experimental evidence.

Our calculated elastic moduli are lower in comparison with experiment, albeit we note that the measured mechanical properties are dependent upon the experimental method; measured values can change by more than $130 \%$.

Accordingly, atomistic models, used to calculate the mechanical properties of a nanoparticle, must include all the microstructural features observed experimentally, such as dislocations and grain boundaries. Simulated amorphisation and re-crystallization enables microstructural features to be captured within the atomistic model - analogous to experiment, they evolve naturally during the crystallization process. For example, a $\Sigma 3$ grain boundary 

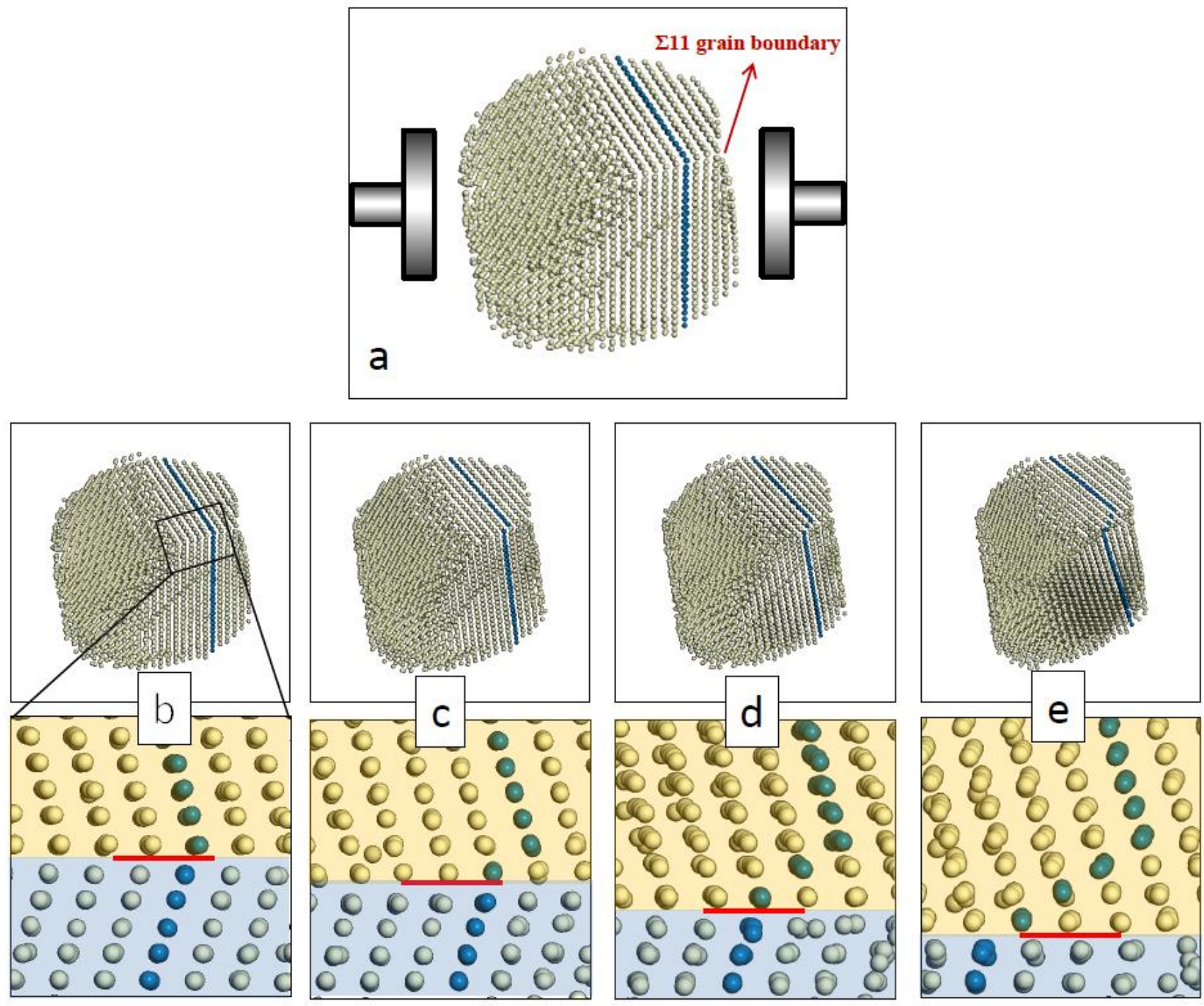

Figure 5 Atomistic structures of the $8 \mathrm{~nm}$ polyhedral NP under uniaxial strain: (a) strain=0.000, (b) strain=0.100, (c) strain $=0.118$, (d) strain $=0.153$, (e) strain=0.186. A segment of the nanoparticle, viewed along $\langle 110\rangle$ at each strain state, reveals more clearly the grain boundary. Indicative of Oswald ripening, the top grain (yellow) consumes the bottom grain (blue); only cerium atoms are shown for clarity.

evolved within the $6 \mathrm{~nm}$ polyhedral nanoparticles, whereas a $\Sigma 11$ grain boundary evolved in the $8 \mathrm{~nm}$ particle

Previously, nanorods with $\Sigma 3$ grain boundaries were calculated to have a compressive yield strength of $10 \mathrm{GPa}^{38}$. Here, we find that $6 \mathrm{~nm}$ NP comprising $\Sigma 3$ grain boundaries have an identical $(10 \mathrm{GPa})$ compressive yield strength, which suggest that the shape is less important than the microstructure. Indeed, the $8 \mathrm{~nm} \mathrm{NP}$, which includes a $\Sigma 11$ grain boundary, has a compressive strength of only $6 \mathrm{GPa}$. 
Wang at $\mathrm{al}^{47}$ performed high pressure angle dispersive $\mathrm{X}$ - Ray diffraction (ADXD) measures on nanocrystalline ceria and they found that nanoceria exhibit negative volume compressibility (NVC) when isotropic pressure is applied using silicon oil as pressure transmitting medium (PTM). The authors explain this effect by considering the nanocrystal to comprise a (crystalline) core - (amorphous) shell system where the amorphous shell undergoes a low density amorphous to high density amorphous (LDH - HDA) transition, above a certain critical pressure. The increase in the applied pressure causes an apparent NVC that is explained if the atoms at the (crystalline) core transform to the amorphous state, increasing the thickness of the amorphous shell.

Our simulations provide evidence to this effect. In particular, imposing uniaxial pressure, we note amorphisation at the surface of the nanoparticles for both cuboidal (Figure S1d) and polyhedral (Figure S2j) shapes.

\section{CONCLUSIONS}

Atomistic models of ceria nanoparticles were generated using simulated amorphisation and re-crystallisation. The models span various sizes $(6-8 \mathrm{~nm})$, shapes (polyhedral, nanocube, spherical), nanoform (core-shell), microstructure (grain-boundaries, dislocations, point defects) and oxidation state (fully oxidised, $\mathrm{CeO}_{2}$, reduced, $\left.\mathrm{CeO}_{1.9}\right)$. The mechanical properties of the nanoparticles were then calculated by simulating each nanoparticle under uniaxial stress.

We find that all attributes (size, morphology, nano-form, microstructure, oxidation state) influence the mechanical properties; grain-boundaries have the greatest impact reducing the bulk modulus and strength by up to $400 \%$ and $200 \%$ respectively.

Our simulations also predict strain-induced Oswald ripening enabling mechanical control over the micro-structure and hence properties of the material 


\section{AUTHOR INFORMATION}

\section{Corresponding author}

Email: D.C.Sayle@kent.ac.uk

\section{ACKNOWLEDGMENTS}

This work was supported by the Engineering and Physical Sciences Research Council (EPSRC) and the School of Physical Sciences, University of Kent.

\section{SUPPORTING INFORMATION}

Potential models and their accuracy; plastic deformation mechanisms of ceria nanoparticles; oxygen disorder at grain-boundaries.

\section{REFERENCES}

1 Ta N.; Liu J.; Shen W. Tuning the shape of ceria nanomaterials for catalytic applications. Cuihua Xuebao/Chinese J. Catalysis, 2013, 34, 838-850.

2 Malvasi L.; Fisher C. A. J.; Islam M. S. Oxide-ion and proton conducting electrolyte materials for clean energy applications: structural and mechanistic features. Chem. Soc. Rev., 2010, 39, 4370-4387;

3 Corma A.; Atienzar P.; García H.; Chane-Ching J. Y. Hierarchically mesostructured doped $\mathrm{CeO} 2$ with potential for solar-cell use. Nature Materials, 2004, 3, 394-397.

4 Di Monte R.; Kašpar J.; Bradshaw H.; Norman C. A rationale for the development of thermally stable nanostructured CeO2-ZrO2-containing mixed oxides. Journal of Rare Earths, 2008, 26, 136-140.

${ }^{5}$ Chen J.; S. Patil, Seal S.; McGinnis J. F. Rare earth nanoparticles prevent retinal degeneration induced by intracellular peroxides. Nature nano-technology, 2006, 1, 142-150. 
${ }^{6}$ Oh M. H.; Nho J. S.; Cho S. B.; Lee J. S.; Singh R. K. Polishing behaviors of ceria abrasives on silicon dioxide and silicon nitride CMP. Powder Technol, 2011, 206, 239-245.

${ }^{7}$ Taniguchi T.; Katsumata K. I.; Omata S.; Okada K.; Matsushita N. Tuning growth modes of ceria-based nanocubes by a hydrothermal method. Crystal Growth and Design, 2011, 11, 3754-3760.

${ }^{8}$ Qian L.; Zhu J.; Du W.; Qian X.; Solvothermal synthesis, electrochemical and photocatalytic properties of monodispersed $\mathrm{CeO} 2$ nanocubes. Mater. Chem. Phys., 2009, 115, 835-840.

9 Bugayeva N.; Robinson J. Synthesis of hydrated $\mathrm{CeO} 2$ nanowires and nanoneedles. Materials Science and Technology, 2007, 23, 237-241.

${ }^{10}$ Feng X.; D. C. Sayle D. C.; Wang Z. L.; Paras M. S.; Santora B.; Sutorik A. C.; Sayle T. X. T.; Yang Y.; Ding Y.; Wang X.; Her Y. -S.; Converting ceria polyhedral nanoparticles into single-crystal nanospheres. Science, 2006, 312, 1504-1508.

11 Yang S.; Gao L.; Controlled synthesis and self-assembly of $\mathrm{CeO} 2$ nanocubes. J. Am. Chem. Soc. 2006, 128, 9330-9331.

12 Cordeiro M. A. L.; Weng W.; Stroppa D. G.; Kiely C. J.; Leite E. R.; High resolution electron microscopy study of nanocubes and polyhedral nanocrystals of cerium(IV) oxide. Chemistry of Materials, 2013, 25, 2028-2034.

${ }^{13}$ Gao F.; Lu Q.; Komarneni S.; Fast synthesis of cerium oxide nanoparticles and nanorods. Journal of Nanoscience and Nanotechnology, 2006, 6, 3812-3819.

${ }^{14}$ Pan C.; Zhang D.; Shi L.; Fang J. Template-free synthesis, controlled conversion, and $\mathrm{CO}$ oxidation properties of $\mathrm{CeO} 2$ nanorods, nanotubes, nanowires, and nanocubes. European Journal of Inorganic Chemistry, 2008, , 2429-2436.

15 Deori K.; Gupta D.; Saha B.; Deka S. Design of 3-dimensionally self-assembled $\mathrm{CeO} 2$ nanocube as a breakthrough catalyst for efficient alkylarene oxidation in water. ACS Catalysis, 2014, 4, 3169-3179

${ }^{16}$ Ishikawa Y.; Takeda M.; TsukimotoS.; Nakayama K. S. ; Asao N. Cerium Oxide Nanorods with Unprecedented Low-Temperature Oxygen Storage Capacity. Adv. Mater. 2015, 28, 1467-1471

17 Pergolesi D.; Fabbri E.; Cook S. N.; Roddatis V.; Traversa E.; Kiliner J. A. Tensile Lattice Distortion Does Not Affect Oxygen Transport in Yttria-Stabilized ZirconiaCeO2 Heterointerfaces. ACS Nano, 2012, 6, 10524-10534 
${ }^{18}$ An J.; Bae J.; Hong S.; Koo B.; Kim Y. B.; Gür T. M.; Prinz F. B. Grain boundary blocking of ionic conductivity in nanocrystalline yttria-doped ceria thin films. Scripta Materialia, 2015, 104, 45-48.

${ }^{19}$ Sayle T. X.; Sayle L. W.; Sayle D. C. Liquid crystal seed nucleates liquid-solid phase change in ceria nanoparticles. Physical Chemistry Chemical Physics, 2015, 17(6), 4441-4447.

${ }^{20}$ Landau M. V.; Vidruk R.; Vingurt D.; Fuks D.; Herskowitz M. Grain boundaries in nanocrystalline catalytic materials as a source of surface chemical functionality. Reviews in Chemical Engineering, 2014, 30(4), 379-401.

${ }^{21}$ Brezesinski T.; Wang J.; Senter R.; Brezesinski K.; Dunn B.; Tolbert S. H. On the correlation between mechanical flexibility, nanoscale structure, and charge storage in periodic mesoporous CeO2 thin films. ACS nano, 2010, 4(2), 967-977.

22 Warren O. L.; Shan Z.; Asif S. S.; Stach E. A.; Morris J. W.; Minor A. M. In situ nanoindentation in the TEM. Materials Today, 2007, 10(4), 59-60.

${ }^{23}$ Shin C.; Jeon I.; Jeon S.; Khim Z. G. Single nanoparticle alignment by atomic force microscopy indentation. Applied Physics Letters, 2009, 94(16), 163107.

${ }^{24}$ Han, X.; Zhang Y.; Zheng K.; Zhang X.; Zhang Z.; Hao Y. J.; Guo J. Y.; Wang Z. L. Low-temperature in situ large strain plasticity of ceramic SiC nanowires and its atomic-scale mechanism. Nano letters, 2007, 7(2), 452-457.

25 Suresh S.; Li J.; Materials science: Deformation of the ultra-strong. Nature, 2007, 456(7223), 716-717.

${ }^{26}$ Lockwood A. J.; Inkson B. J. In situ TEM nanoindentation and deformation of Sinanoparticle clusters. Journal of Physics D: Applied Physics, 2008, 42(3), 035410.

27 Guo D.; Xie G.; Luo J. Mechanical properties of nanoparticles: basics and applications. Journal of Physics D: Applied Physics, 2013, 47(1), 013001.

28 Chueh W.C.; McDaniel A.H.; Grass M.E.; Hao Y.; Jabeen N.; Liu Z.; Haile S.M.; McCarty K.F.; Bluhm H.; El Gabaly F.; Highly enhanced concentration and stability of reactive $\mathrm{Ce} 3+$ on doped $\mathrm{CeO} 2$ surface revealed in operando. Chemistry of Materials, 2012, 24(10), 1876-1882.

${ }^{29}$ Sayle T. X. T.; Parker S. C.; Catlow C. R. A. The role of oxygen vacancies on ceria surfaces in the oxidation of carbon monoxide. Surface Science, 1994, 316(3), 329336.

30 Catlow C. R. A.; Freeman C. M.; Royle R. L. "Recent studies using static simulation techniques." Physica B+C, 1985, 131, 1, 1-12. 
${ }^{31}$ Smith W.; Forester T. R. DL_POLY_2. 0: a general-purpose parallel molecular dynamics simulation package. Journal of molecular graphics, 1996, 14(3), 136141.

${ }^{32}$ Sayle D.C.; Seal S.; Wang Z.; Mangili B.C.; Price D.W.; Karakoti A.S.; Kuchibhatla S.V.; Hao Q.; Mobus G.; Xu X.; Sayle T.X. Mapping nanostructure: a systematic enumeration of nanomaterials by assembling nanobuilding blocks at crystallographic positions. ACS nano, 2008, 2(6), pp.1237-1251.

${ }^{33}$ Sayle T. X. T.; Ngoepe P. E.; Sayle D. C. Generating structural distributions of atomistic models of Li2O nanoparticles using simulated crystallisation. Journal of Materials Chemistry, 2010, 20(46), 10452-10458.

${ }^{34}$ Humphrey W.; Dalke; A.; Schulten K. VMD: visual molecular dynamics. Journal of molecular graphics, 1996, 14(1), 33-38.

35 Sayle T. X. T.; Parker S. C.; Sayle D. C.; Shape of CeO 2 nanoparticles using simulated amorphisation and recrystallisation. Chemical communications, 2004, (21), 2438-2439.

${ }^{36}$ Cormack A. N.; Lamphier S.; Wang B.; Gubb T.; Reed K. Simulations of ceria nanoparticles. In Proc. R. Soc. A, 2015, 471, 20150218

37 Sayle D. C.; Feng X.; Ding Y.; Wang Z. L.; Sayle T. X. "Simulating synthesis": ceria nanosphere self-assembly into nanorods and framework architectures. Journal of the American Chemical Society, 2007. 129(25), 7924-7935.

${ }^{38}$ Sayle T.X.; Inkson B.J.; Karakoti A.; Kumar A.; Molinari M.; Möbus G.; Parker S.C.; Seal S.; Sayle D.C.; Mechanical properties of ceria nanorods and nanochains; the effect of dislocations, grain-boundaries and oriented attachment. Nanoscale, 2011, 3(4), pp.1823-1837.

${ }^{39}$ Bhatta U.M.; Reid D.; Sakthivel T.; Sayle T.X.; Sayle D.; Molinari M.; Parker S.C.; Ross I.M.; Seal S.; Möbus G.; Morphology and surface analysis of pure and doped cuboidal ceria nanoparticles. The Journal of Physical Chemistry C, 2013, 117(46), 24561-24569.

${ }^{40}$ Tan J.P.Y.; Tan H.R.; Boothroyd C.; Foo Y.L.; He C.B.; Lin M.; Three-dimensional structure of $\mathrm{CeO} 2$ nanocrystals. The Journal of Physical Chemistry C, 2011, 115(9), 3544-3551.

${ }^{41}$ Grimmer H.; Bollmann W.T.; Warrington; D.H.; Coincidence-site lattices and complete pattern-shift in cubic crystals. Acta Crystallographica Section A: Crystal Physics, Diffraction, Theoretical and General Crystallography, 1974, 30(2), 197207. 
42 Hansen T.W.; DeLaRiva A.T.; Challa S.R.; Datye A.K. Sintering of catalytic nanoparticles: particle migration or Ostwald ripening?. Accounts of chemical research, 2013, 46(8), pp.1720-1730.

${ }^{43}$ Guo X.; Waser R. Electrical properties of the grain boundaries of oxygen ion conductors: acceptor-doped zirconia and ceria. Progress in Materials Science, 2006, 51(2), 151-210.

${ }^{44}$ Landau M.V.; Vidruk R.; Vingurt D.; Fuks D.; Herskowitz M.; Grain boundaries in nanocrystalline catalytic materials as a source of surface chemical functionality. Reviews in Chemical Engineering, 2014, 30(4), 379-401.

${ }^{45}$ Ye F.; Yin C.Y.; Ou D.R; Mori T. Relationship between lattice mismatch and ionic conduction of grain boundary in YSZ. Progress in Natural Science: Materials International, 2014, 24(1). 83-86.

${ }^{46}$ Wang Y.; Duncan K.; Wachsman E. D.; Ebrahimi F. The effect of oxygen vacancy concentration on the elastic modulus of fluorite-structured oxides. Solid State lonics, 2007, 178, 53-58

47 Wang Q.; He D.; Peng F.; Lei L.; Liu P.; Shuai Y.; Wang P.; Xu C.; Liu J. Unusual compression behaviour of nanocrystalline CeO2. Scientific Reports, 2014, 4:4441.

48 Sato K.; Yugami H.; Hashida T. Effect of rare-earth oxides on fracture properties of ceria ceramics. Journal of Materials Science, 2004, 39(18), 57655770.

49 Sato K.; Suzuki K.; Yashiro K.; Kawada T.; Yugami H.; Hashida T.; Atkinson A.; Mizusaki J.; Effect of $\mathrm{Y} 2 \mathrm{O} 3$ addition on the conductivity and elastic modulus of (CeO 2) 1- x (YO 1.5) x. Solid State lonics, 2009, 180(20), 1220-1225.

${ }^{50}$ Rodenbough P.P.; Song J.; Walker D.; Clark S.M.; Kalkan B.; Chan S.W.; Size dependent compressibility of nano-ceria: Minimum near $33 \mathrm{~nm}$. Applied Physics Letters, 2015, 106(16), 163101.

51 Nakajima A.; Yoshihara A.; Ishigame M. Defect-induced Raman spectra in doped CeO2. Physical Review B, 1994, 50(18), 13297. 